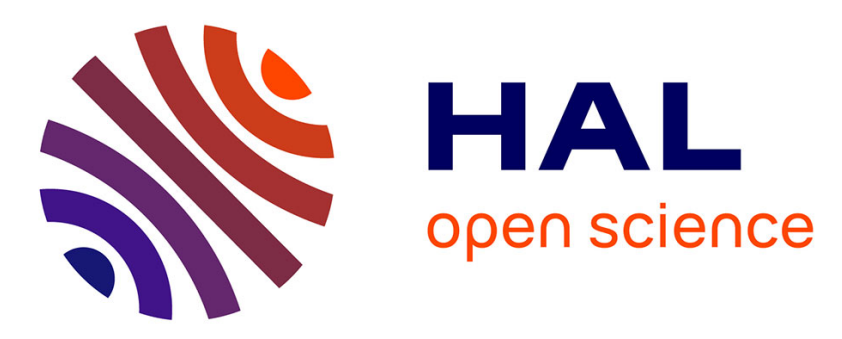

\title{
Mathematics of Pharmacokinetics and Pharmacodynamics: Diversity of Topics, Models and Methods
}

Gennady Bocharov, Anas Bouchnita, Jean Clairambault, Vitaly Volpert

\section{- To cite this version:}

Gennady Bocharov, Anas Bouchnita, Jean Clairambault, Vitaly Volpert. Mathematics of Pharmacokinetics and Pharmacodynamics: Diversity of Topics, Models and Methods. Mathematical Modelling of Natural Phenomena, 2016. hal-01413795

\section{HAL Id: hal-01413795 \\ https://hal.inria.fr/hal-01413795}

Submitted on 11 Dec 2016

HAL is a multi-disciplinary open access archive for the deposit and dissemination of scientific research documents, whether they are published or not. The documents may come from teaching and research institutions in France or abroad, or from public or private research centers.
L'archive ouverte pluridisciplinaire HAL, est destinée au dépôt et à la diffusion de documents scientifiques de niveau recherche, publiés ou non, émanant des établissements d'enseignement et de recherche français ou étrangers, des laboratoires publics ou privés. 


\title{
Mathematics of Pharmacokinetics and Pharmacodynamics: Diversity of Topics, Models and Methods
}

\author{
G. Bocharov ${ }^{1}$, A. Bouchnita ${ }^{2,3,6,7}$, J. Clairambault ${ }^{4,5}$, V. Volpert $6,7,8$ \\ ${ }^{1}$ Institute of Numerical Mathematics of the Russian Academy of Sciences, Gubkina Street 8, Moscow, Russia \\ ${ }^{2}$ LBBE, UMR 5558 CNRS, University Lyon 1, 69622 Villeurbanne, France \\ ${ }^{3}$ LERMA, Mohammadia School of Engineering, University Mohamed V, Rabat, Morocco \\ ${ }^{4}$ INRIA Team Mamba, INRIA Paris, 2 rue Simone Iff, CS 42112, 75589 Paris, France \\ ${ }^{5}$ Sorbonne Universités, UPMC, Lab. J.-L. Lions UMR 7598 CNRS, 4, pl. Jussieu, b. c. 187, 75252 Paris, France \\ ${ }^{6}$ Institut Camille Jordan, UMR 5208 CNRS, University Lyon 1, 69622 Villeurbanne, France \\ ${ }^{7}$ INRIA Team Dracula, INRIA Antenne Lyon la Doua, Villeurbanne, Lyon, France \\ ${ }^{8}$ Laboratoire Poncelet, UMI 2615 CNRS, Bolshoy Vlasyevskiy Pereulok 11, 119002 Moscow, Russia
}

\begin{abstract}
Keywords and phrases: pharmacokinetics-pharmacodynamics, mathematical models, model analysis, parameter estimation, computer simulation
\end{abstract}

Mathematics Subject Classification: 92C30, 92C45

\begin{abstract}
A short review on pharmacokinetics-pharmacodynamics (PK-PD) presented below aims to show the evolution of some concepts and ideas in this field. Some of them are developed in more detail in the papers of this issue. The key question for a practical application of PK-PD models is the ability to estimate the model parameters using patients data. In [1] a novel approach to an accurate quantification of the uncertainty in parameter estimates attributed to inter-individual variability is proposed. The analyzed PK-PD model is formulated as a compartmental ODE system. The methodology of recognizing and capturing the uncertainty in predicted quantities of interest due to inter-individual variability when the individual is not available for repeated measurements may prove to be invaluable in the risk assessment of future experiments and drug applications. Anticancer molecular PK-PD in a cell population dynamics model with drug delivery optimisation is discussed in [2]. The works [3] and [4] deal with various aspects of hemostasis modelling, and [5] with metabolic aspects in a whole-body setting. These studies represent the diversity of aspects of PK-PD modelling nowadays: theoretical about parameter estimation in general versus applied to medical questions in particular, localised cell population versus whole-body settings, cell population and whole-body versus patient population settings.
\end{abstract}

\section{1. "Spatial dimension" of PK-PD problems in immunology}

The classical modelling approach in PK-PD is based on mathematical models representing a compartmental view of the organism [6]. The models consider organs and cells as compartments in which the concentration of a drug is spatially uniform or homogeneous. However, recent clinical and experimental studies of infections such as human immunodeficiency virus (HIV) or simian immunodeficiency virus (SIV) clearly indicate that the view has to be extended to consider a genuine spatial dimension of the tissues and organs in which the infection and drug distribution processes take place.

One of the first evidences of the key role of spatial distribution of drugs in the efficacy of antiretroviral (ART) HIV treatment was presented in [7]. It was shown that HIV continues to replicate in lymphatic tissues of some in- 
dividuals under taking ART regimens considered fully suppressive, based on undetectable viral loads in peripheral blood. One mechanism proposed to explain the persistent replication of HIV in lymphatic tissues was lower concentrations of the ART drugs in those tissues compared with peripheral blood. Overall, a clear association between low drug concentrations in tissues and persistent viral replication was documented. The evidence has been further corroborated in recent studies of the persistent productive HIV infection during therapy [8] and SIV infections in elite controllers [9]. The existence of the drug-free zones in lymphoid tissues is broadly discussed in [10]. It is a remarkable finding that multiple drug concentrations in lymph nodes can be about 100-times lower than those in blood for the same patient [7].

To analyse the novel aspects of the drug dynamics and effect in organs spatially extended models of drug transport in tissues are needed. For example, the drug concentration field can be studied using the reaction-diffusion equation:

$$
-\operatorname{div}(\mathbb{D} \nabla c(x))+\alpha c(x)=\sum_{k=1}^{M} \rho \delta\left(x-x_{k}\right),
$$

where $\mathbb{D}$ is a tensor diffusion coefficient, $\mathbb{D}=\mathbb{D}^{(i)}$ in domains $\Omega_{i}, i=1, \ldots, N$, specifying various structures of the organs under investigation. The equation is understood in a weak sense. Although the above model is rather simple, it can be used in conjunction with the anatomical description of the tissue structure to provide novel insights into the spatial patterns of drug distribution in complex organ environments. Instructive example is provided by studies of type I interferon (IFN) distribution in lymph nodes (LNs) [11, 12]. The structural organisation of LNs is quite elaborate [13] and a paradigmatic view gives a simplified but still a representative geometry of the $\mathrm{LN}$. The 3D geometry of a paradigmatic LN approximated with a tetrahedral mesh is shown in Figure 1, left. The elements considered include subcapsular sinus (SCS), trabecular sinuses, B-cell follicles, T cell zone, conduits. This geometry requires a multiple length scale resolution for the representation of the conduits and other major domains. The above reaction-diffusion partial differential equation governing the interferon dynamics was used to analyse the steady-state distribution of IFN across the subdomains of the 3D lymph node. The LN domain $\Omega$ consists of subdomains represesnting SCS $\Omega_{1}$, trabecular sinuses $\Omega_{2}$, conduit "network" $\Omega_{3}$, B cell follicle $\Omega_{4}$, T cell zone $\Omega_{5}$. Obviously, the penetration of the cytokine to various parts of the $\mathrm{LN}$ is governed by the relative balance of convection, diffusion and degradation/absorption of the IFN molecules. Under these settings, $M$ is the number of cell producing IFN, $x_{k}$ denotes the cell location, $\rho$ is the production rate, $\alpha$ the degradation/absorbtion rate of IFN. There is a non-homogeneity in diffusion coefficients $\mathbb{D}^{(1,2,3)}=\mathbb{D}^{*}, \mathbb{D}^{(4)}=0.1 \mathbb{D}^{*}, \mathbb{D}^{(5)}=0.01 \mathbb{D}^{*}$ as suggested by experimentally observed differences of small molecular tracers distribution in LN domains [14]. The regions of LNs that contain T lymphocytes (the cortical and para-cortical zones or the $\Omega_{5}$ region) are characterised by weak molecular diffusion, which is associated with poor hydraulic conductivity [14]. Calibrating the model parameters using the estimates from $[15,16] \mathbb{D}^{*}=d \mathbb{I}, d=0.16 \mathrm{~mm}^{2} / \mathrm{hr}, \rho=4.4 \cdot 10^{-4} \mathrm{pg} / \mathrm{hr}, \alpha=0.012 / \mathrm{hr}$ allows one to examine the extent of non-homogeneity of the IFN field in $\mathrm{LN}$ as shown in Figure 1, centre. The numerical solution for the model predicts that a substantial variation of IFN concentration takes place within the LN, i.e. by two orders of magnitude. Therefore the compartmentalisation of the cytokine can occur due to the diffusion process. The conclusion is robust with respect to variations in geometrical structure of $\mathrm{LN}$ and justified variations in the model parameter values. Type I interferon is generally considered as an antiviral drug for HIV infection. In a broader context, it can be hypothesised that when the drug distributions is concerned the morphology and biophysical properties of secondary lymphoid organs can lead to the formation of poorly protected areas. In these areas, localization of the cells infected by viruses, such as CD4+ T lymphocytes or macrophages in the case of HIV infection provides the conditions for the continuation of active infectious processes.

The effect of spatial compartmentalisation of this important cytokine in lymphoid tissue is predicted based on embedding a reaction-diffusion model into a spatial 3D context of the $\mathrm{LN}$ and some basic information about the hydraulic conductivity taken into account. Deeper insights into the spatial patterns of cytokine and drug penetration into LN will require a finer approximation of the LN structures, e.g., such as the conduit network and the blood microvascular system as well as studies of the molecular transport in domains densely packed with cells. Figure 1, right shows a 3D model of the $\mathrm{LN}$ with geometrically and topologically consistent representation of fibroblastic reticular cell network. Some elements necessary to move in this direction has already been elaborated in recent studies of fluid flow through LNs [17-20]. 

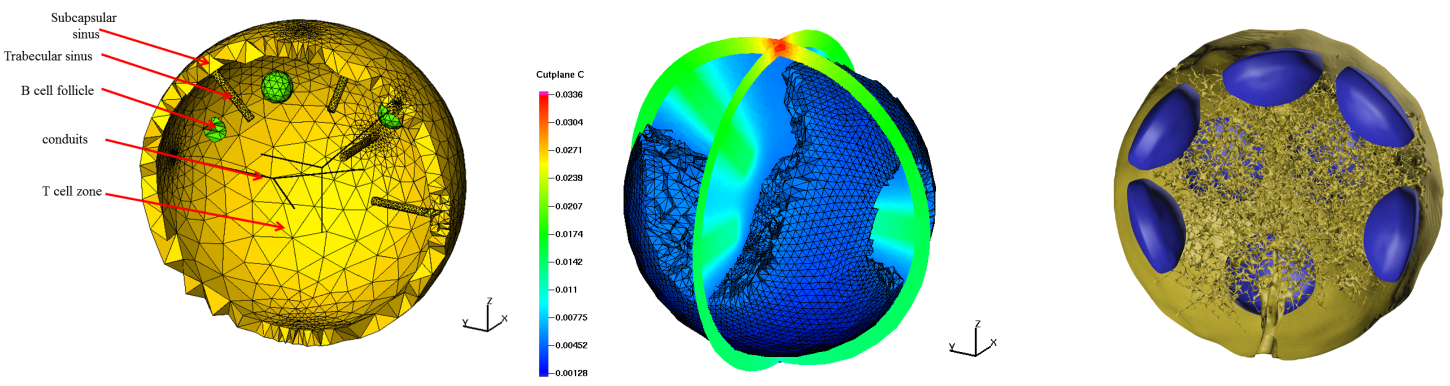

FIGURE 1. Spatial organisation of lymph node (LN). Left: Finite-element based approximation of major structural elements of a paradigmatic LN. Centre: 3D reaction-diffusion model predictions of the spatial distribution of type I interferon in LN. Color bar reflects the relative concentration of the molecules. Right: Geometric model of LN with anatomically consistent representation of the B-cell follicles and Fibroblastic Reticular Cell network.

\section{Non-spatial models in oncology}

Representing healthy and cancer proliferating cell populations, the targets of anticancer drugs, may involve space, but most often the spatial structure of the cell populations is not known. Tumours are far from presenting themselves as spatially well-organised tissues; furthermore anticancer drugs act on molecular or functional targets in cell populations, that are by nature highly heterogeneous in physiology as well in pathology. Space is thus not necessarily relevant to describe their action, whose result is seen on cell population numbers or growth rates. Much more relevant than space-structured models are physiologically structured models, that are amenable to account for the relevant biological variability (=heterogeneity) of the cell population under study.

In this special issue, an age-structured transport model of the cell division cycle in healthy and cancer cell populations is presented, following previous ones [21-24]. Its originality is that it is coupled with an ODE model for the PK-PD of the two main drugs used in colorectal cancer: 5-fluorouracil and oxaliplatin. It has been originally designed in the framework of chronotherapeutics (chrono-PK-PD) [25], but it can also be used outside of this framework, provided that the time differences in gating at the cell cycle checkpoints between healthy and cancer cell populations are preserved.

Given such fundamental differences, one can, as shown in the article of the present issue, design optimised strategies for the delivery of anticancer drugs. The optimisation method proposed operates under the constraint of preserving the healthy cell population. Another crucial aspect of theoretical therapeutic optimisation consists in avoiding the emergence of resistant cell subpopulations, which is the other major pitfall of therapeutics in oncology. It is only alluded at in this article, but it is studied in detail in [26, 27]. In the models used, cell populations are structured in a phenotype representing the expression of drug resistance in the cells. They are basically nonspatial, but space can be introduced as complementary structure variable when it is relevant to the description of the heterogeneity under study, for instance if the tumour is assumed to be spheroid-shaped [27]. Then PK-PD ODE models for the drug considered (a cytotoxic and a cytostatic) could be coupled to the phenotype-structured models as it has been done in age-structured models of the cell cycle, but this remains to be done.

\section{PK-PD and systems pharmacology in blood clotting pathologies}

Developing mathematical models of blood coagulation is essential in understanding the pathogenesis of blood clotting abnormalities such as thrombosis and hemophilia. Usually, the developed models are firstly built in order to describe the dynamics of healthy blood coagulation. However, it is sufficient to change some parameters associated with a coagulation disorder to describe pathological blood coagulation. The ultimate goal of these models is to study the action of treatments on the coagulation process. Despite the wide range of mathematical methods used in the development of PK-PD hemostasis models, the modellers are faced with numerous challenges due to the complexity of the blood clotting system. Below, we present a state of the art of the approaches used in the mathematical modelling of blood clotting disorders and their treatment.

Venous thrombosis is a blood clotting disorder characterized by the formation of excessive blood clots within a vein resulting in the perturbation of blood flow. Warfarin and heparin are two anticoagulant drugs that are usually 
prescribed to prevent venous thrombosis [28]. In a recent model [29], the effect of warfarin on clot growth in flow was studied. Warfarin is an anti-vitamin $\mathrm{K}$ drug which downregulates the synthesis of some clotting factors in the liver. Its action is monitored by an in vitro assay called INR (international normalized ratio) which is based on the necessary time for prothrombin conversion into thrombin. In order to prevent thrombosis, the INR should be maintained between 2.0 and 3.0 during warfarin treatment. The authors used previously developed models for both in vivo clot formation and in vitro PT and INR assays, and then compared the action of warfarin on individual patients under these two conditions. They conducted numerical simulation describing clot growth during warfarin treatment under various flow conditions. They concluded that the in vitro assays do not represent a reliable tool for monitoring warfarin treatment due to the absence of blood flow in in vitro tests. This explains the persistence of thrombosis for some patients even when their INR is in the targeted range.

In addition to warfarin, heparin is one of the commonly used venous thrombosis treatments. Heparin upregulates the activity of antithrombin which reduces blood coagulability. In a recent work [30], Dydek and Chaikof equipped a previously developed model for in situ clot growth with the action of heparin. The resulting model is detailed and focuses on the spatio-temporal dynamics of clot growth during heparin treatment. The authors evaluated the effects of different doses and types of heparin under venous and arterial flows and included the effect of circulating tissue factor. In the future, this work can be combined with pharmacokinetics models of heparin treatments to assess the efficacy and safety of various protocols.

A comprehensive model which encompasses both the PK-PD and the in vitro assays aspects of venous thrombosis was developed using systems pharmacology [31]. It uses ODEs to describe the concentrations of drugs, proteins, and clotting factors in different time scales which affect each other. In this work, the authors compared the results of different in vitro tests and clotting times such as PT and aPTT during anticoagulant treatment. They studied the action of different drugs such as warfarin and heparin and simulated the pathogenesis of blood clotting disorders such as thrombosis, hemophilia A and B. To the best of our knowledge, this is one of the few complex models that were validated by experimental data in the literature. The model can serve as an in silico predicting tool in drug development and clinical trials.

Although most of the PK-PD models of thrombosis study the action of warfarin and heparin, some of the recent works are devoted to the action of new oral anticoagulants such as rivaroxaban. It is a direct factor Xa inhibitor which is commonly used to prevent strokes and thrombo-embolic events in adult patients. An important PK-PD model which assesses the efficacy and safety rivaroxaban treatments was developed by Burghaus et al. [32]. This work is of practical interest because it is based on previously validated models [33, 34]. Furthermore, it encompasses both the extrinsic and intrinsic pathways as well as the effects of blood flow on clot growth. All of these factors make the model appropriate for clinical studies. Recently, the model was used in order to study the optimal dosing schedule for switching from warfarin to rivaroxaban treatment (Burghaus et al. 2014). Another systems pharmacology model of rivaroxaban action was developed by Zhou et al. [36]. It contains one of the most complete descriptions of the blood coagulation cascade and accurately predicts clotting times during anticoagulant treatment. Direct thrombin inhibitors are another class of new oral anticoagulant drugs which are used in the prevention of thromboembolic events. Dabigatran etexilate is one of the commonly used direct thrombin inhibitors as it can replace warfarin but does not require monitoring by the INR test. The only in vitro assay which can potentially assess the efficacy and safety of such treatments is the thrombin generation test. In a recent study [37], the authors used a calibrated version of this test to assess the efficacy of dabigatran. Although the study does not directly provide a mathematical model which can be used in in silico dabigatran treatments assessment, it presents valuable experimental data which can help the future developments of PK-PD models describing the action of dabigatran on the coagulation process.

There are many other systems pharmacology models which describe blood coagulation pathologies and the action of treatments ([38-42]). While most of these models are more appropriate for an accurate multi-scale description of the coagulation process during treatment, they are usually built in complex and sophisticated ways that incorporate many details. As a result, it becomes difficult to interpret the obtained results and to derive simple relations between the parameters of interest. To encourage the use of multi-scale models in clinical studies, Gulati et al. showed how to reduce a 62-state model to 5-state model [39]. They also derived mechanistic relationships between coagulation parameters that agree with experimental data.

Developing PK-PD models of blood coagulation pathologies requires an appropriate characterization of individual patientsṕrofiles. Brummel-Ziedins [43] addressed this point by developing and validating computational approaches which capture the dynamics of thrombin generation in individual patients depending on plasma composition. Such approaches can be used in identifying thrombosis risk during anticoagulant treatments. Other models 
study the hemostatic response in individuals with pre-existing health conditions associated with clotting disorders including rheumatoid arthritis [44], cancer [45], and deep venous thrombosis [46].

PK-PD models of thrombosis and other blood coagulation disorders represent a promising tool that can potentially predict individual haemostatic responses. However, for these models to be incorporated in clinical studies, it is crucial to confront them with in vitro and in vivo blood clotting experimental data. Ultimately, we can expect that accurate computer models of blood coagulation will replace in vitro diagnostic assays. Until then, a significant effort should be made in order to develop more accurate and experimentally validated models that capture all the pathophysiological aspects of blood coagulation.

\section{Future developments}

\subsection{Whole-body and multi-scale modelling}

The development of patient-specific drug therapies requires high-resolution mathematical models for assimilating of the vast amount of data including -omics, sequencing, flow cytometry, imaging, immune profiling characterizing the organism response to drug intervention at various levels of resolution. Therapies of some complex pathologies also include adoptive immune cell transfer in addition to drug injections. This combination of therapeutic agents of different nature call for the models considering the spatial distribution of target cells (e.g., CD4 T cells in follicles in HIV infection) and the migration of immune cells in various parts of the LN (e.g., CTL destroying infected cells). Overall, a shift from a compartmental consideration of the PK-PD processes to multi-physics and multiscale framework (see, e.g. [47]) in active and soft tissues and organs in a 3D setting environment suggests the following agenda for research:

1. Parameterised computational 3D models of the organs and tissues viewed as idealised 3D structures complementing the reconstruction based approaches from imaging data;

2. Anatomically based mathematical models of processes dynamics;

3. Systemic cell recirculation in the host in conjunction with local migration within organs and tissues;

4. Biophysical properties of tissues, e.g., hydraulic conductivity, porosity;

5. Blood and lymph flow patterns in lymphoid organs;

6. Transport and distribution governing equations (e.g., sub-, normal or super-diffusion) of cytokines, chemokines, drugs;

7. Fluid-tissue interactions;

8. Regulation of structural homeostasis in health and disease (e.g., the role of FRCs in immune responses).

As space becomes the current frontier in systems immunology [48], technologies to observe and analyze cells and drugs distributions in tissues are developing. Implementation of a systems approach to PK-PD problems requires looking at the structure, dynamics, regulation and design principles of specific organs. Whereas the dynamics have been extensively studied within the compartmental framework, the last three topics require further investigation. This should help to overcome the concern existing in PK-PD community about the impact of mathematical modelling and simulation [49,50]. Linking the structure of organs to well established PK-PD modelling methodologies in a computationally tractable and physiologically relevant manner will require cross-disciplinary research teams. "This is not the end, it is not even the beginning of the end, but it is perhaps the end of the beginning." (Sir Winston Churchill Speech "The End Of The Beginning", 20th November 1942).

\subsection{Drug resistance, or how to deal with the failure of targeted therapies in cancer}

Targeted therapies in cancer aim at controlling known hubs in intracellular signalling pathways. The cases of imatinib in Chronic Myelogenous Leukaemia (CML) [51] and of All-transretinoic acid in Acute Promyelocytic Leukaemia [52] are known success stories in targeted therapies, however such cases remain rare because most often when a hub is blocked in a cancer cell, other hubs may take over, and one such cell is enough to thrive into an emergent drug-resistant subpopulation. The fundamental heterogeneity in phenotype of cancer cell populations [53], that has been proposed to be the result of some sort of bet hedging [54] and that can be accurately represented in phenotype-structured models [55], may thus explain the evolution towards drug resistance in tumour cell populations. To document such mechanisms, PK-PD models to describe the fate of drugs in healthy and cancer cells are not enough. Indeed, one must consider the levels of expression of all concerned genes, as has been recently described for instance in [56] for resistance to doxorubicin in Mutiple Myeloma. In this way, the future prospects that are open to model such phenomena in cancer go far beyond PK-PD and offer an immense challenge 
to mathematicians: how to model and analyse the action of drugs in cancer, considering issues of both toxicity in healthy tissues and of emergence of drug resistance in tumour cell populations? This will involve designing relevant functional phenotypes from whole genome dynamic data processing. Some bioinformatic methods are already available for this, but the relevant corresponding mathematical models are still to be designed, and new and advanced statistical methods to process biological data will also be needed. When one considers the necessity to design on the basis of such models optimal control algorithms for therapeutic strategies to be clinically efficient, the challenge appears even more tremendous. But it has to be met.

Acknowledgements. The research of GB was funded by the Russian Science Foundation (Grant 15-11-00029).

\section{References}

[1] H.T. Banks, R. Baraldi, J. Catenacci, N. Myers. Parameter estimation using unidentified individual data in individual based models. Math. Model. Nat. Phenom., 11 (2016), no. 6,

[2] J. Clairambault, O. Fercoq. Physiologically structured cell population dynamic models with applications to combined drug delivery optimisation in oncology. Math. Model. Nat. Phenom., 11 (2016), no. 6,

[3] A. Bouchnita, K. Bouzaachane, T. Galochkina, P. Kurbatova, P. Nony, V. Volpert. An individualized blood coagulation model to predict INR therapeutic range during warfarin treatment. Math. Model. Nat. Phenom., 11 (2016), no. 6,

[4] A. Modepalli Susree, B. Mohan Anand. Reaction mechanisms and kinetic constants used in mechanistic models of coagulation and fibrinolysis. Math. Model. Nat. Phenom., 11 (2016), no. 6,

[5] T.O. Shepelyuk, M.A. Panteleev, A.N. Sveshnikova. Computational modeling of quiescent platelet energy metabolism in the context of whole-body glucose turnover. Math. Model. Nat. Phenom., 11 (2016), no. 6,

[6] P. Macheras, A. Iliadis, Modeling in Biopharmaceutics, Pharmacokinetics and Pharmacodynamics, Homogeneous and Heterogeneous Approaches. Springer, (2006), 293-308.

[7] C.V. Fletcher, K. Staskus, S.W. Wietgrefe, M. Rothenberger, C. Reilly, J.G. Chipman, G.J. Beilman, A. Khoruts, A.Thorkelson, T.E. Schmidt, J. Anderson, K. Perkey, M. Stevenson, A.S. Perelson, D.C. Douek, A.T. Haase, T.W. Schacker. Persistent HIV-1 replication is associated with lower antiretroviral drug concentrations in lymphatic tissues. Proc Natl Acad Sci U S A, 111 (2014), no. 6, $2307-2312$.

[8] R. Lorenzo-Redondo, H.R. Fryer, T. Bedford, E.Y. Kim, J. Archer, S.L. Kosakovsky Pond, Y.S. Chung, S. Penugonda, J.G. Chipman, C.V. Fletcher, T.W. Schacker, M.H. Malim, A. Rambaut, A.T. Haase, A.R. McLean, S.M. Wolinsky. Persistent HIV-1 replication maintains the tissue reservoir during therapy. Nature, 530 (2016), no. 7588, 51-56.

[9] Y. Fukazawa, R. Lum, A.A. Okoye, H. Park, K. Matsuda, J.Y. Bae, S.I. Hagen, R. Shoemaker, C. Deleage, C. Lucero, D. Morcock, T. Swanson, A.W. Legasse, M.K. Axthelm, J. Hesselgesser, R. Geleziunas, V.M. Hirsch, P.T. Edlefsen, M. Piatak Jr, J.D. Estes, J.D. Lifson, L.J. Picker. B cell follicle sanctuary permits persistent productive simian immunodeficiency virus infection in elite controllers .Nat Med. 21 (2015), no. 2, 132-139.

[10] A. Licht, G. Alter. A drug-free zone-lymph nodes as a safe haven for HIV. Cell Host Microbe, 19 (2016), no. 3, $275-276$.

[11] G. Bocharov, A. Danilov, Yu. Vassilevski, G.I. Marchuk, V.A. Chereshnev, B. Ludewig. Reaction-diffusion modelling of interferon distribution in secondary lymphoid organs. Math. Model. Nat. Phenom., 6 (2011), no. 7, 13-26.

[12] G.A. Bocharov, A.A. Danilov, Y.V. Vassilevski, G.I. Marchuk, V.A. Chereshnev, B. Ludewig. Simulation of the interferon-mediated protective field in lymphoid organs with their spatial and functional organization taken into consideration. Doklady Biological Sciences 439 (2011), no. 1, 194-196.

[13] T. Junt, E. Scandella, B. Ludewig. Form follows function: lymphoid tissue microarchitecture in antimicrobial immune defence. Nature Reviews Immunology. 8 (2008), 764-775.

[14] T. Lammermann, M. Sixt. The microanatomy of T-cell responses. Immunological Reviews, 221 (2008), $26-43$.

[15] G. Bocharov, R. Züst, L. Cervantes-Barragan, T. Luzyanina, E. Chiglintsev, V.A. Chereshnev, V. Thiel, B. Ludewig. A systems immunology approach to plasmacytoid dendritic cell function in cytopathic virus infections. PLoS Pathogens, 6 (2010), no. 7, e1001017.

[16] J. Keener, J. Sneyd. Mathematical Physiology. Springer-Verlag, New York, 2009.

[17] R. Savinkov, A. Kislitsyn, D.J. Watson, R. van Loon, I. Sazonov, M. Novkovic, L. Onder, G. Bocharov. Data-driven modelling of the FRC network for studying the fluid flow in the conduit system. Engineering Applications of Artificial Intelligence, 2016, http://dx.doi.org/10.1016/j.engappai.2016.10.007

[18] M. Jafarnejad, M.C. Woodruff, D.C. Zawieja, M.C. Carroll, J.E. Jr moore. Modeling lymph flow and fluid exchange with blood vessels in lymph nodes. Lymphatic research and biology, 13 (2015), no. 4, 234-247.

[19] A. Kislitsyn, R. Savinkov, M. Novkovic, L. Onder, G. Bocharov. Computational approach to 3D modeling of the lymph node geometry. Computation, 3 (2015), 222-234.

[20] L.J. Cooper, J.P. Heppell, G.F. Clough, B. Ganapathisubramani, T. Roose. An image-based model of fluid flow through lymph nodes. Bull. Math. Biol., 78 (2016), no. 1, 52-71.

[21] F. Billy, J. Clairambault. Designing proliferating cell population models with functional targets for control by anti-cancer drugs. Discrete and Continuous Dynamical Systems - Series B, 18 (2013), no. 4, 865-889.

[22] F. Billy, J. Clairambault, F. Delaunay, C. Feillet, N. Robert. Age-structured cell population model to study the influence of growth factors on cell cycle dynamics. Mathematical Biosciences and Engineering, 10 (2013), no. 1, 1-17.

[23] F. Billy, J. Clairambault, Q. Fercoq. Optimisation of cancer drug treatments using cell population dynamics. In: A. Friedman, E. Kashdan, U. Ledzewicz, H. Schättler (eds.) Mathematical Models and Methods in Biomedicine, Lecture Notes on Mathematical Modelling in the Life Sciences, 265-309. Springer, New York, 2013.

[24] F. Billy, J. Clairambault, O. Fercoq, S. Gaubert, T. Lepoutre, T. Ouillon, S. Saito. Synchronisation and control of proliferation in cycling cell population models with age structure. Mathematics and Computers in Simulation, 96 (2014), 66-94.

[25] J. Clairambault. Deterministic mathematical modelling for cancer chronotherapeutics: cell population dynamics and treatment optimisation. In "Mathematical Oncology 2013", A. d'Onofrio and A. Gandolfi Eds., Part III, 265-294, Birkh'auser, New York, 2014. 
[26] A. Lorz, T. Lorenzi, M.E. Hochberg, J. Clairambault, B. Perthame. Populational adaptive evolution, chemotherapeutic resistance and multiple anti-cancer therapies. ESAIM: Mathematical Modelling and Numerical Analysis, 47 (2013), no. 2, $377-399$.

[27] A. Lorz, T. Lorenzi, J. Clairambault, A. Escargueil, B. Perthame. Modeling the effects of space structure and combination therapies on phenotypic heterogeneity and drug resistance in solid tumors. Bull. Math. Biol., 77 (2015), no. 1, 1-22.

[28] B. Dahlback. Haematology: blood coagulation. The Lancet 355 (2000), 1627-1632.

[29] L. de Pillis, E.J. Graham, K. Hood, Y. Ma, A. Radunskaya, J. Simons. Injury-initiated clot formation under flow: a mathematical model with warfarin treatment. In: Applications of Dynamical Systems in Biology and Medicine, 75-98. Springer New York, 2015.

[30] E.V.Dydek, E.L. Chaikof. Simulated thrombin generation in the presence of surface-bound heparin and circulating tissue factor. Annals of Biomedical Engineering, 44 (2016), no. 4, 1072-1084.

[31] T. Wajima, G.K. Isbister, S.B. Duffull. A comprehensive model for the humoral coagulation network in humans. Clinical Pharmacology and Therapeutics, 86 (2009), no. 3, 290-298.

[32] R. Burghaus, K. Coboeken, T. Gaub, L. Kuepfer, A. Sensse, H.U. Siegmund, J. Lippert. Evaluation of the efficacy and safety of rivaroxaban using a computer model for blood coagulation. PLoS One, 6 (2011), no. 4, e17626.

[33] S.D. Bungay, P.A. Gentry, R.D. Gentry. A mathematical model of lipid-mediated thrombin generation. Mathematical Medicine and Biology, 20 (2003), no. 1, 105-129.

[34] M.F. Hockin, K.C. Jones, S.J. Everse, K.G. Mann. A model for the stoichiometric regulation of blood coagulation. Journal of Biological Chemistry, 277 (2002), no. 21, 18322-18333.

[35] R. Burghaus, K. Coboeken, T. Gaub, C. Niederalt, A. Sensse, H.U. Siegmund, J. Lippert. Computational investigation of potential dosing schedules for a switch of medication from warfarin to rivaroxaban-an oral, direct Factor Xa inhibitor. Frontiers in Physiology, 5 (2013), 417-417.

[36] X. Zhou, D.R.H. Huntjens, R.A.H.J. Gilissen. A systems pharmacology model for predicting effects of factor Xa inhibitors in healthy subjects: assessment of pharmacokinetics and binding kinetics. CPT: Pharmacometrics \& Systems Pharmacology, 4 (2015), no. 11, 650659.

[37] Gribkova, I. V., Lipets, E. N., Rekhtina, I. G., Bernakevich, A. I., Ayusheev, D. B., Ovsepyan, R. A., ... \& Sinauridze, E. I. (2016). The modification of the thrombin generation test for the clinical assessment of dabigatran etexilate efficiency. Scientific Reports, 6 . ISO 690

[38] L. Cromme, H. Völler, F. Gäbler, A. Salzwedel, U. Taborski. Computer-aided dosage in oral anticoagulation therapy using phenprocoumon. H'amostaseologie, 30 (2010), no. 4, 183-189.

[39] A. Gulati, G.K. Isbister, S.B. Duffull. Scale reduction of a systems coagulation model with an application to modeling pharmacokineticpharmacodynamic data. CPT: Pharmacometrics \& Systems Pharmacology, 3 (2014), no. 1, 1-8.

[40] D. Luan. Computational modeling and simulation of thrombus formation. Doctoral dissertation, Cornell University, 2009.

[41] L.D. Lynd, B.J. O’ Brien. Advances in risk-benefit evaluation using probabilistic simulation methods: an application to the prophylaxis of deep vein thrombosis. Journal of Clinical Epidemiology, 57 (2004), no. 8, 795-803.

[42] L.A. Parunov, O.A. Fadeeva, A.N. Balandina, N.P. Soshitova, K.G. Kopylov, M.A. Kumskova, M. A. Panteleev. Improvement of spatial fibrin formation by the anti-TFPI aptamer BAX499: changing clot size by targeting extrinsic pathway initiation. Journal of Thrombosis and Haemostasis, 9 (2011), no. 9, 1825-1834.

[43] K. Brummel-Ziedins. Models for thrombin generation and risk of disease. Journal of Thrombosis and Haemostasis, 11 (2013), no. s1, 212-223.

[44] A. Undas, M. Gissel, B. Kwasny-Krochin, P. Gluszko, K.G. Mann, K.E. Brummel-Ziedins. Thrombin generation in rheumatoid arthritis. dependence on plasma factor composition. Thrombosis and Haemostasis, 104 (2010), no. (2), 224-230.

[45] L.E. Clegg, F. Mac Gabhann. Systems biology of the microvasculature. Integrative Biology, 7 (2015), no. 5, $498-512$.

[46] K.E. Brummel-Ziedins, C.Y. Vossen, S. Butenas, K.G. Mann, F.R. Rosendaal. Thrombin generation profiles in deep venous thrombosis. Journal of Thrombosis and Haemostasis, 3 (2015), no. 11, 2497-2505.

[47] A. Bouchnita, G. Bocharov, A. Meyerhans, V. Volpert. Hybrid approach to model the spatial regulation of T cell responses. BMC Immunology, (2016) in press.

[48] B. Ludewig, J.V. Stein, J. Sharpe, L. Cervantes-Barragan, V. Thiel, G. Bocharov. A global “imaging” view on systems approaches in immunology. Eur J Immunol. 42 (2012), no. 12, 3116-3125.

[49] S.R. Allerheiligen. Impact of modeling and simulation: myth or fact? Clin. Pharmacol. Ther., 96 (2014), no. 4, $413-415$.

[50] P.L. Bonate. What happened to the modeling and simulation revolution? Clin. Pharmacol. Ther., 96 (2014), no. 4, $416-417$.

[51] B.J. Druker, M. Talpaz, D.J. Resta, B. Peng, E. Buchdunger, J.M. Ford, N.B. Lydon, H. Kantarjian, R. Capdeville, S. Ohno-Jones, C.L. Sawyers. Efficacy and safety of a specific inhibitor of the BCR-ABL tyrosine kinase in chronic myeloid leukemia. N. Engl. J. Med., 344 (2001), no. 14, 1031-1037.

[52] T. Haferlach. Molecular genetic pathways as therapeutic targets in AML. In: Educational book, ASH 2008 meeting, 400-411, 2008.

[53] R.H. Chisholm, T. Lorenzi, J. Clairambault. Cell population heterogeneity and evolution towards drug resistance in cancer: biological and mathematical assessment, theoretical treatment optimisation. Biochimica et Biophysica Acta, 1860 (2016), $2627-2645$.

[54] B.Brutovsky, D. Horvath. Structure of intratumor heterogeneity: Is cancer hedging its bets? arXiv, 1307.0607, 2013.

[55] R.H. Chisholm, T. Lorenzi, A. Lorz, A.K. Larsen, L.N. Almeida, A. Escargueil, J. Clairambault. Emergence of drug tolerance in cancer cell populations: an evolutionary outcome of selection, nongenetic instability, and stress-induced adaptation. Cancer Res., 75 (2015), no. 6, 930-939.

[56] A. Wu, Q. Zhang, G. Lambert, Z. Khin, R.A. Gatenby, H.J. Kim, N. Pourmand, K. Bussey, P.C. W. Davies, J.C. Sturma, R.H. Austin. Ancient hot and cold genes and chemotherapy resistance emergence. Proc. Nat. Acad. Sci. USA 112 (2015), no. 33, 10467-10472. 